\title{
A new astronomical facility for Peru: transforming a telecommunication's 32-metre antenna into a radio-telescope
}

\author{
José Ishitsuka ${ }^{1,2}$, M. Ishitsuka**, N. Kaifu*, M. Inoue ${ }^{2}$, M. Tsuboi $^{3}$, \\ M. Ohishi ${ }^{2}$, T. Kondo ${ }^{4}$, Y. Koyama ${ }^{4}$, T. Kasuga ${ }^{6}$, K. Fujisawa $^{5}$, \\ K. Miyazawa*, M. Miyoshi ${ }^{2}$, T. Umemoto ${ }^{3}$, T. Bushimata ${ }^{2}$, \\ S. Horiuchi ${ }^{7}$ and E. Vidal ${ }^{1}$ \\ ${ }^{1}$ Instituto Geofísico del Perú, \\ Calle Badajoz 169, Urb. Mayorazgo IV Etapa, Ate-Vitarte, Lima 3, Perú \\ email: pepe@geo.igp.gob.pe \\ ${ }^{2}$ National Astronomical Observatory of Japan, 2-21-1 Osawa, Mitaka, Tokyo 181-8588, Japan \\ ${ }^{3}$ Nobeyama Radio Observatory, Minamimaki, Minamisaku, Nagano 384- 1305, Japan \\ ${ }^{4}$ National Institute of Information and Communications Technology 893-1 Hirai, Kashima, \\ Ibaraki 314, Japan \\ ${ }^{5}$ Yamaguchi University, Yoshida 1677-1, Yamaguchi 753-8512, Japan \\ ${ }^{6}$ Hosei University, Kajinocho 3-7-2, Koganei, Tokyo 184-8584, Japan \\ ${ }^{7}$ Swinburne University of Technology, Faculty of Information and Communication \\ Technologies, PO Box 218, Hawthorn VIC 3122, Australia \\ ${ }^{*}$ Retired from NAOJ, ${ }^{* *}$ Retired from IGP
}

\begin{abstract}
In 1984 an INTELSAT antenna of $32 \mathrm{~m}$ of diameter was constructed at 3370 metres above the sea level on the Peruvian Andes. At the time Entel Perú the Peruvian telecommunications company managed the antenna station, of almost 12 hectares in extension. In 1993 the government transferred the station to the private telecommunications company Telefónica del Perú. Since transoceanic fiber optics replaced radically satellite communications in 2002, a beautiful 32- metre parabolic antenna was finished its tele-communications mission and become available for other use. So in cooperation with the National Astronomical Observatory of Japan we began coordination to transform the antenna into a radio-telescope.

Researches on interstellar medium around Young Stellar Objects (YSO) are possible using methanol maser that emits at $6.7 \mathrm{GHz}$, so initially we will monitor and survey maser sources at the southern sky. An ambient temperature receiver with $T_{\mathrm{rx}}=60 \mathrm{~K}$ was developed at Nobeyama Radio Observatory and is ready to be installed. The antenna will be controlled by the Field System FS9 software installed in a PC within a Linux environment. An interface between antenna and PC was developed at Kashima Space Research Center in Japan by Mr E. Vidal.

In the near future S-band $(2 \mathrm{GHz}), \mathrm{X}$-band $(8 \mathrm{GHz}), 12 \mathrm{GHz}$ and $22 \mathrm{GHz}$ observations are planned.

The peculiar position and altitude of the Peruvian Radio Observatory will be useful for VLBI observations with the VLBA for astronomical observation and geodetic measurements. For Peru where few or almost non astronomical observational instruments are available for research, implementation of the first radio observatory is a big step to foster sciences at graduate and postgraduate levels of universities.

Worldwide tele-communications antennas recently tend to finish their role as tele-communications antennas. Several of them are transformed into useful observational instruments.
\end{abstract}

Keywords. Maser, Peru, radio-astronomy, YSO 


\section{Introduction}

To perform astronomical observations in developing countries, where astronomy faculties at universities are not yet established, such a $32 \mathrm{~m}$ diameter radio-telescope would be an excellent means to develop astronomy and basic sciences. Around the world some successful cases are working well, one in Ceduna of the University of Tasmania, Australia and another in Japan, a 32-m radio-telescope at Yamaguchi City managed by Yamaguchi University.

In Peru Nippon Electric Company (NEC) constructed in 1984 a 32-m parabolic antenna for satellite communication managed by the past telephone company Entel Peru, later in 1993 government transferred the antenna station to the private company Telefónica del Perú. Then in 2000 the Peruvian INTELSAT communication station stopped operations. Two years later, we began conversations with telephone company in order to transfer the antenna to Instituto Geofísico del Perú and to transform into a radio-telescope. Transfer of the antenna ownership from the Telefónica del Perú to the Instituto Geofísico del Perú is under negotiation.

\section{Science and education}

Since methanol masers around high-mass star forming regions are good tracers to investigate early evolutionary phase of YSO, methanol masers appear to be closely associated with newly formed massive stars (Menten 2002; Minier et al. 2003 and references therein); initially we are planning to monitor and survey methanol maser sources.

There are 65 universities in Peru, 28 are public and 37 are private, 14 of the 65 universities have faculty of physics. The new radio-telescope facility will become an important instrument to educate undergraduate students, also will allow perform researches of astrophysics for graduate students. Running a radio-telescope involves antenna technologies, receiver system, down converters, computers and software development, students of other fields would be also educated and trained. We can settle radio-astronomy in Peru putting in operation the 32-m antenna.

\section{Collaborations}

From the beginnings of our project, National Astronomical Observatory of Japan and National Institute of Information and Communications Technology strongly encouraged us to develop instruments and train our staff. Collaborations made possible to develop and construct equipments to be installed on the radio-telescope. That also made possible to train and educate students, scientists, and engineers that will use in the future the radio-telescope.

\section{6.7-GHz receiver}

Peruvian antenna used to transmit at $6 \mathrm{GHz}$ and receive at $4 \mathrm{GHz}$ for tele-communications, so connecting a $6.7 \mathrm{GHz}$ receiver to the transmission feed will be the most efficient way to transform the antenna into a radio-telescope. We can receive $6.7 \mathrm{GHz}$ methanol maser line spectrum signals, with $70 \mathrm{MHz}$ of band width adding continuum observations.

A $6.7-\mathrm{GHz}$ ambient temperature receiver have been prepared at Nobeyama Radio Observatory (Figure 1) and successfully tested in laboratory, from HOT-COLD load receiver temperature $T_{\mathrm{rx}}$ was $60 \mathrm{~K}$, then we tested the receiver installing on a 20-metre radio-telescope of VERA Project (Sasao 1996; Honma et al. 2000) and could get W3(OH) methanol spectrum (Figure 2). 


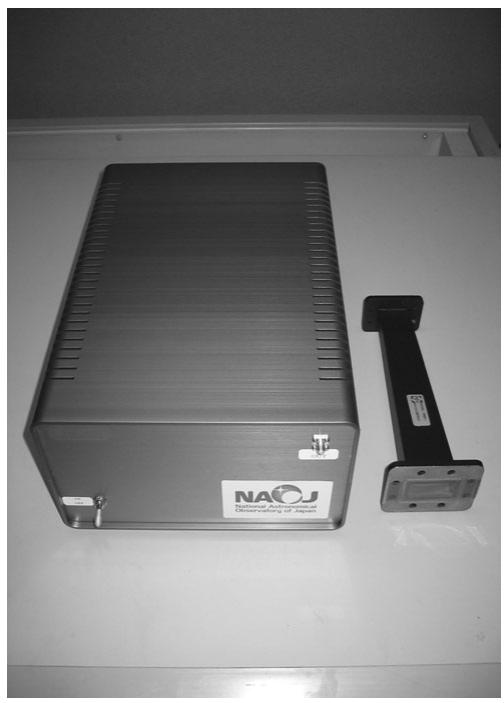

Figure 1. Ambient temperature $6.7 \mathrm{GHz}$ receiver, $T_{\mathrm{rx}}=60 \mathrm{~K}$. Designed and built in Nobeyama Radio Observatory of NAOJ

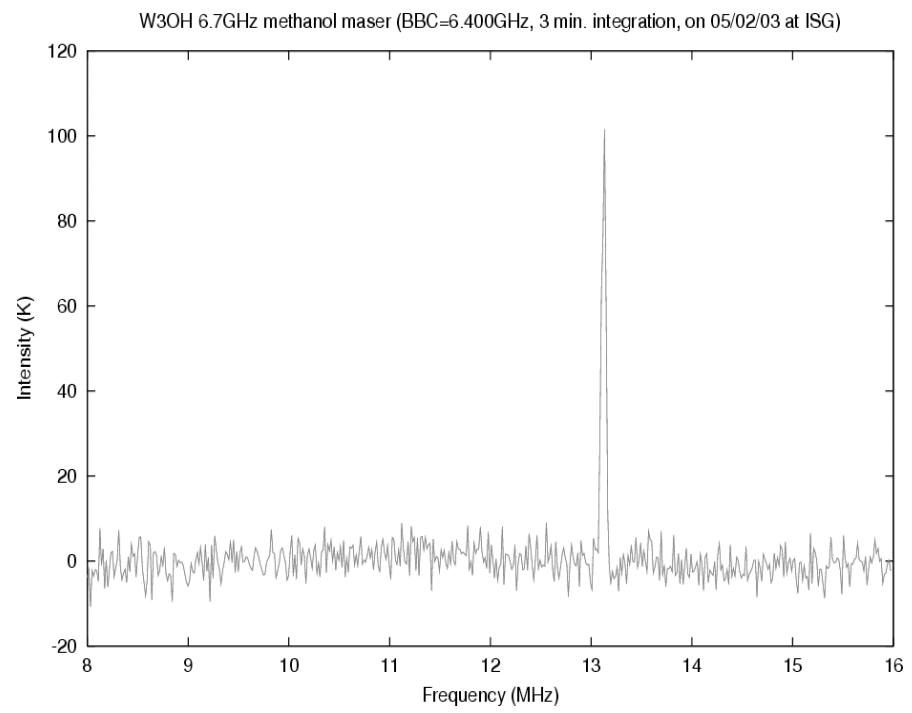

Figure 2. Methanol maser line spectrum of W3(OH)

\section{Antenna control system}

Being controlled by the Field System FS9 developed by NASA, the telescope is capable of not only single dish observations but also VLBI observations with other telescopes located in different places.

An interface was developed in Kashima Space Communication Center, see Figure 3 it will allow control the radio-telescope from a PC equipped with Linux Debian. Field System FS9 is the software that commands the Antenna Control Unit (ACU) to move the radio-telescope appropriately and perform astronomical observations. 


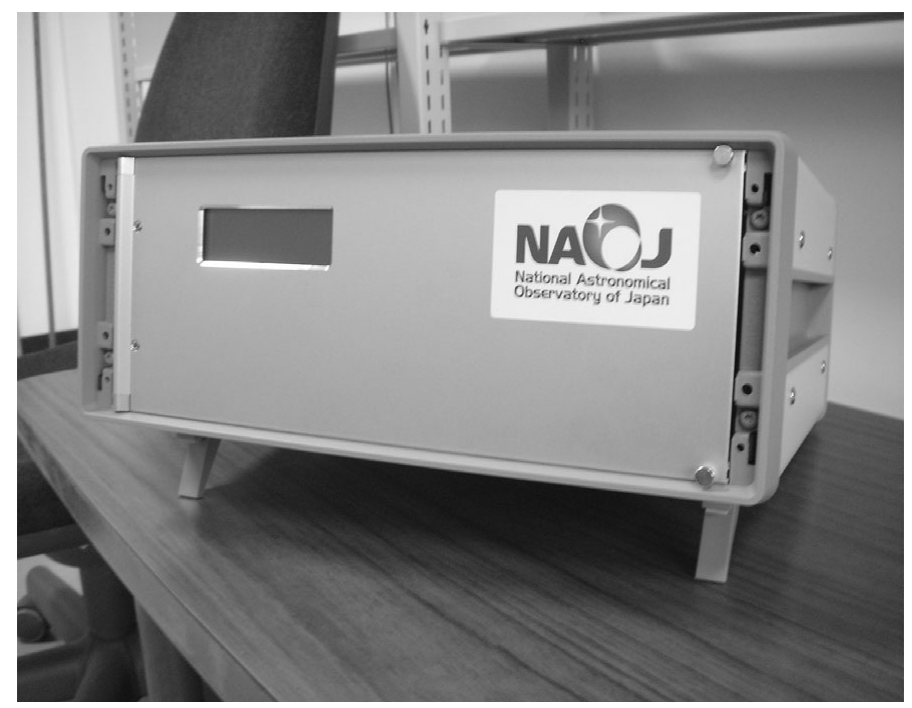

Figure 3. Interface between $\mathrm{PC}(\mathrm{FS} 9)$ and $\mathrm{ACU}$ of antenna.

\section{Future frequencies and VLBI}

Once the Peruvian radio-telescope is tuned to receive $6.7 \mathrm{GHz}$ signals, in the future we are planning to implement other frequencies receivers, such as $2 \mathrm{GHz}$ and $8 \mathrm{GHz}$ to perform Geodetic VLBI observations, and $12.2 \mathrm{GHz}$ and $22 \mathrm{GHz}$ to observe in VLBI mode, observations with other international VLBI arrays are in the scope, include Peruvian radio-telescope, as a VLBA station will improve observations for VLBA, especially for low elevation sources (Horiuchi et al., 2005).

\section{Location}

The Telecommunications Antenna Station is located over a hill in a beautiful valley called Valle del Mantaro, at $3370 \mathrm{~m}$ above the see level and have a comfortable dry weather. Station used to be called Estacion Terrena de Sicaya.

The coordinates are: Latitude: $-12^{\circ} 02^{\prime} 15^{\prime \prime}$, Longitude: $-75^{\circ} 17^{\prime} 39^{\prime \prime}$

The location is peculiar and interesting to perform geodesy observations, precise measurements of the antenna location will be useful to measure plate tectonics on the region.

\section{Conclusions}

Re-use of tele-communication antennas as a radio-telescope is one of the way to develop and settle astronomy in countries where lack of observational instruments is a serious problem.

Implementing the first radio observatory in Peru is a big step and foster sciences at graduate and postgraduate levels of universities.

Collaboration of developed countries to develop instruments and educate people are vital. Equipment or financial assistance for developing countries are important to start this kind of project. In the mean time scholarships to train and educate people must be implemented.

International collaborations have to be improved, participation of people of Peruvian radio-telescope in scientific events will level up skills and knowledge in radio astronomy, related sciences and technologies. 


\section{Acknowledgements}

We acknowledge to each of the staff at Kashima Space Communication Research Center, thanks to them now we have an antenna control system ready to be installed. We also acknowledge to people in Japan that contribute with donations and made possible to have an emergency found to support running costs of our radio-telescope.

\section{References}

Horiuchi S., Murphy D. W., Ishitsuka J.K. \& Ishitsuka M. 2005, ASOP p572

Honma M., Kawaguchi N. \& Sasao T. 2000, SPIE Vol. 4015, Radio Telescope p624-p631

Menten K. M. 2002, IAU Symp. 206, From Protostars to Black Holes 125

Minier V., Ellingsen S. P., Norris R. P. \& Booth R. S. 2003, A\&A, Vol. 403 p205

Sasao T. 1996, 4th APT Workshop p94-p104

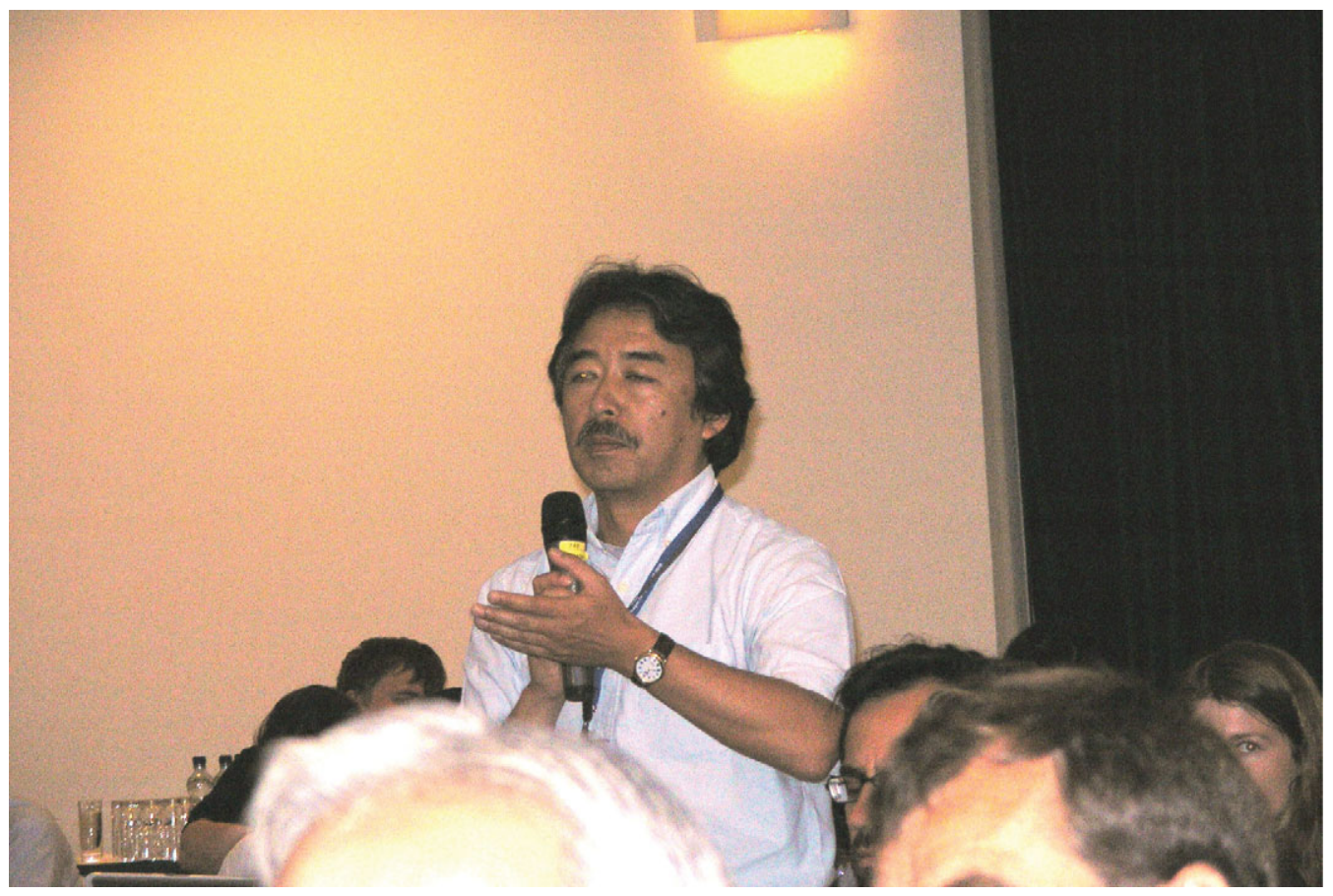

José Ishitsuka 


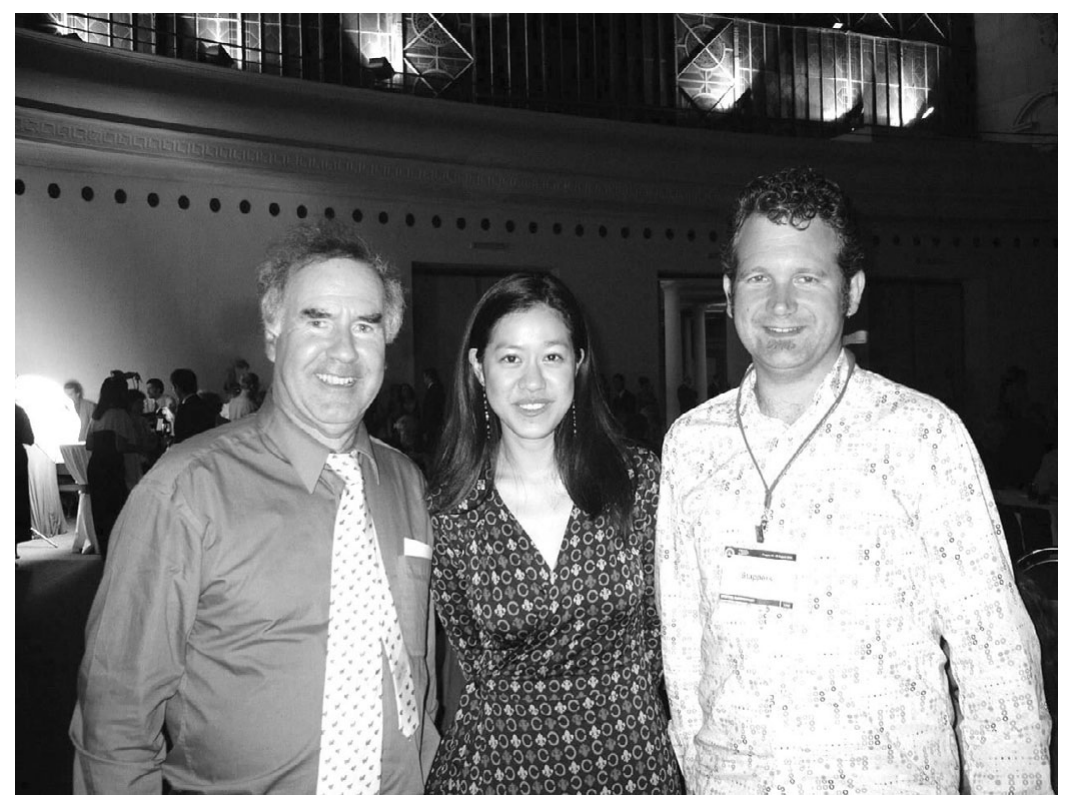

left to right: John Hearnshaw, Siramas Komonjinda and Ben Stappers

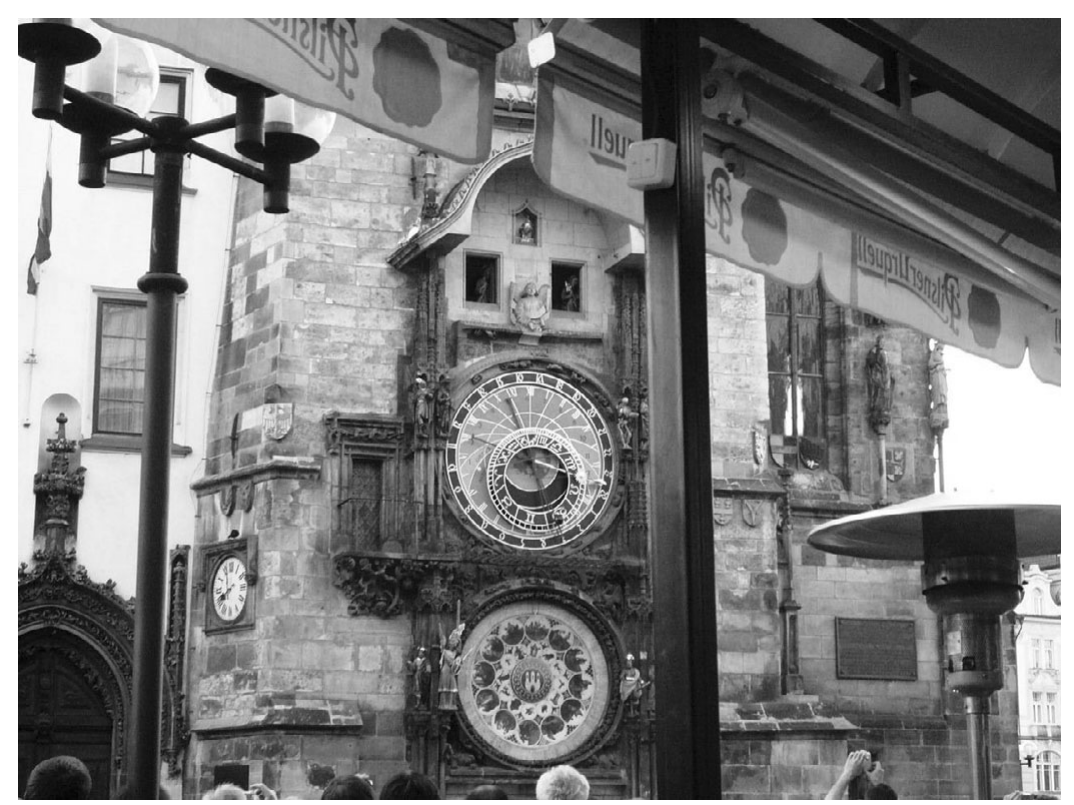

The Astronomical Clock, Old Town City Hall, Prague 